\title{
Theoretical Investigation of Ultrathin Gate Dielectrics
}

\author{
ALEXANDER A. DEMKOV ${ }^{\mathrm{a}, *}$, XIAODONG ZHANG $^{\mathrm{b}}$ and HEATHER LOECHELT ${ }^{\mathrm{c}}$ \\ ${ }^{a}$ Physical Sciences Research Labs, Motorola, Inc., Tempe, AZ 85284, USA; ${ }^{\mathrm{b}}$ Department of Physics \\ and Astronomy, Ohio University, Athens, $\mathrm{OH} 45701$; ' ${ }^{\mathrm{D}}$ Department of Mathematics, \\ Arizona State University, Tempe, AZ 85287
}

\begin{abstract}
We describe a theoretical methodology for screening potential gate dielectric materials. A recently proposed method for constructing realistic structural models of the Sidielectric interface is used to generate the $\mathrm{Si}-\mathrm{SiO}_{2}-\mathrm{Si}$ and $\mathrm{Si}-\mathrm{SiON}-\mathrm{SiO}_{2}-\mathrm{Si}$ model metaloxide-semiconductor (MOS) structures. We discuss methods to estimate the valence band discontinuity at the corresponding interface. We use Landauer's ballistic transport approach to investigate the low bias leakage through these ultrathin dielectric layers.
\end{abstract}

Keywords: Gate dielectrics; Electronic structure; Quantum transport; Oxynitrides

\section{INTRODUCTION}

The International Technology Roadmap for Semiconductors (ITRS) indicates that the strategy of scaling complimentary metal-oxide-semiconductor (CMOS) devices will come to an abrupt end around the year 2012 [1]. The main reason for this will not be the lithography resolution problems as was anticipated, but rather the leakage current through the silicon dioxide gate with a thickness below $2 \mathrm{~nm}$. In other words, the insulation of the oxide layer breaks down for a very thin layer [2]. The end of $\mathrm{SiO}_{2}$ as a gate insulator spurred an active search for an alternative gate dielectric. Finding such a material has proven to be far from trivial.

There are four principle requirements that a successor gate material should meet. (1) The leakage problem is rooted in the continuous reduction of the gate dielectric's thickness necessary to maintain the capacitance of the MOS structure as its lateral dimensions are being scaled. Therefore a material with a dielectric constant higher than that of $\mathrm{SiO}_{2}$ (approximately four) is sought. (2) The new material should be stable in contact with $\mathrm{Si}$ (that is, not to form silicides or silicates) at temperatures up to $900^{\circ} \mathrm{C}$. This is required to maintain the integrity of a sharp interface during the CMOS processing, such as, e.g., the implant anneal. (3) The density of interfacial traps should be below $10^{12} \mathrm{~cm}^{-2}$ to be on par with $\mathrm{SiO}_{2}$. (4) The conduction band offset should be at least $1 \mathrm{eV}\left(3.2 \mathrm{eV}\right.$ at the $\mathrm{Si}_{-} \mathrm{SiO}_{2}$ interface) to provide a sufficient energy barrier necessary for the gate action at the operational bias.

*Corresponding author. Tel.: 480-755-5016, Fax: 480-755-5055, e-mail: alex.demkov@motorola.com 
Within the adiabatic approximation the dielectric constant of a solid has two major contributions: the electronic and lattice susceptibilities. The orientational term important in polar liquids and molecular solids may be neglected for a covalent or ionic material. The electronic susceptibility scales approximately as a square of the ratio of the plasma frequency $\omega_{p}$ (measures the electron density) over the so called Penn gap $\mathrm{E}_{P G}$ (measures the average energy gap of the electronic spectrum) [3]:

$$
\varepsilon=1+\left(\frac{\hbar \omega_{p}}{E_{P G}}\right)^{2}
$$

With $\mathrm{E}_{P G}$ being about $4-6 \mathrm{eV}$ for most semiconductors, and $\hbar \omega_{p}$ being in the range of $15-17 \mathrm{eV}$, it is clear that it is unlikely that the electronic component alone allows to achieve a dielectric constant much higher than approximately 15 . That is why the vast majority of novel gate dielectrics proposed to this day, e.g., $\mathrm{SrTiO}_{3}$ [4-6], $\mathrm{ZrO}_{2}$ [7], $\mathrm{HfO}_{2}$ [8], $\mathrm{TiO}_{2}$ [9], or $\mathrm{Ta}_{2} \mathrm{O}_{5}$ [10], are insulators that display the high lattice polarizability in the crystalline form due to a soft phonon mode. These materials, however, present a different set of challenges. First and foremost, one needs to suppress the oxidation of Si. Secondly, the thermodynamic stability in contact with $\mathrm{Si}$ is a problem [10]. Third, in most transition metal oxides the bottom of the conduction band is derived from the d-states of the transition metal which are close in energy to the conduction band of Si. This results in a small or negative conduction band offset [11]. In addition, the dielectric constant is typically reduced in the oxide thin films, and there are indications that this reduction is caused by the softmode hardening [12]. This may have severe implications for the feasibility of achieving a high dielectric constant, particularly in amorphous films.

The empirical search for a new gate dielectric is rather costly, and theoretical modeling could prove to be very helpful. In particular, theory provides the information on the chemical bonding, structure, and stability of the Si-dielectric interface, and band offsets. An estimate of the low bias leakage current through the gate, and how it is affected by the atomic structure would be of great interest in this ongoing investigation. In this paper we present a possible methodology that may be used to guide the experimental effort. Using a theoretical atomic level $\mathrm{Si}-\mathrm{SiO}_{2}$ model system, we demonstrate how the band offset may be computed. We study the role of nitrogen at the interface. We investigate the leakage current through an ultra-thin MOS structure using the quantum transmission probability that when intergrated produces an I-V characteristics. A combination of the density functional quantum molecular dynamics (QMD) and ballistic transport theory are employed. To our knowledge, this is the first entirely first principles theoretical analysis of this problem.

\section{PRELIMINARY THEORETICAL CONSIDERATIONS}

We now discuss our approach to the leakage current calculations in nanoscale devices such as ultrathin MOS structures. As the size of the electronic devices continues to shrink, and the computational power of the electronic structure methods steadily increases, we now can compute quantum mechanically both the atomic structure of a very small device and its transport characteristics such as an I-V curve. The electronic structure methods allow to perform predictive calculations for systems containing hundreds and even thousands of atoms. This translates into a few nanometer length scale, and we could take advantage of a simple ballistic transport theory [13]. In this theory a current calculation is reduced to an effective one-dimensional scattering problem. For example, the leakage through a MOS structure is described as tunneling through a potential barrier [14]. The use of a ballistic description is justified for systems with dimensions below the physically important scattering lengths such as the Fermi length, mean free path, and the 
phase relaxation length which in a typical semiconductor are $10 \mathrm{~nm}, 30 \mu \mathrm{m}, 1 \mu \mathrm{m}$, respectively. Transport through $\mathrm{SiO}_{2}$ relevant to our case has been studied theoretically $[15,16]$ and experimentally [17], and the mean free path is reported to be between 0.6 and $1.5 \mathrm{~nm}$, with the LO-phonon emission being a dominant energy-loss mechanism. Therefore, for a $1 \mathrm{~nm}$ thick oxide layer one can use Landauer's formalism. The current is given by:

$$
I_{L R}=\frac{e}{\pi \hbar} \int_{\mu_{2}}^{\mu_{1}} d E T(E) d E
$$

where the $\mu_{1}$ and $\mu_{2}$ are the chemical potentials of the left and right leads, respectively. Thus the central problem is to calculate the barrier transmission function $T(E)$. The transmission probability is often calculated within the WKB approximation. To do so, one needs to know the barrier thickness, height, and the carrier's effective mass $\mathrm{m}^{*}$ [18]. Choosing a proper effective mass for the barrier region appears somewhat difficult. A wide range of values for the "tunneling" mass is used in the literature $[19,20]$. The small parameter in the Luttinger-Kohn theory that allows the use of the envelope function and the effective mass itself is of the order of $\left(a / a_{i}\right)^{2}$, where $a$ is the lattice constant, and the $a_{i}$ is the extent of the "defect" state [21], which in our case is the oxide thickness. When the thickness of the gate dielectric (the scatter) is only $1 \mathrm{~nm}$ the effective mass approximation does not hold (the lattice constant of $\mathrm{Si}$ is $0.535 \mathrm{~nm}$ ). Therefore, the band structure-derived effective mass may be used only with extreme care at this length scale.

We will describe two complementary methods of estimating the band offset at the interface, that gives the barrier height. For the transport calculations we follow the approach of Fisher and Lee which relates the barrier transmission to the Green's function for the scatering region [22]. We generate an atomistic model of the oxide layer, which will be referred to as "defect" to represent a scattering region. Two semi-infinite perfect $\mathrm{Si}$ regions with the same cross section as the "defect", representing the leads are considered attached on both sides of the "defect" structure. The problem is infinite in three dimensions. Applying periodic boundary conditions in two transverse directions we reduce it to a $1 \mathrm{D}$ propagation in the direction normal to the interface. The local orbital basis (see below) allows us to use the Löwdin representation of the Hamiltonian, and the geometry of the effective 1D problem is reflected in the matrix. The semi-infinite leads are treated via corresponding self-energy operators related to the lead's Green's function. This is calculated using a slab-recursion method [23]. Due to the finite range of the basis orbitals the slab-slab coupling in the leads and the defect-lead coupling are treated exactly. In our formulation neither the effective mass nor the electron velocity appear explicitly in the formalism. Instead, a spectral density operator, which can be defined regardless of the system size, plays their role.

Structural calculations have been performed by using a local orbital first principles quantum molecular dynamics (QMD) method designed for applications to large systems and implemented in the Fireball package [24]. The method employs density functional theory within the local density approximation and hard norm-conserving pseudopotentials. The simplified energy functional is a self-consistent generalization of that due to Harris. The total electron density is approximated by a sum of densities of fragments, e.g., atoms that are not necessarily neutral. The method is entirely real space (except in a simple Ewald summation). A short-range non-orthogonal local orbital basis of Fireball orbitals of Sankey and Niklweski offers a good variational flexibility combined with a significant computational advantage [25]. Integrals over the Brillouin zone are evaluated using the special $k$-points of Monkhorst and Pack. If the local energy minimum is sought a fictitious friction force is introduced to guide the system to the minimum energy configuration. Numerous recent applications of the technique to a vareity of materials problems are reviewed in Ref. [26]. 


\section{STRUCTURE FROM QUANTUM MOLECULAR DYNAMICS}

We have theoretically built several model MOS structures. As a starting point we use theoretical structures of the $\mathrm{Si}^{-} \mathrm{SiO}_{2}$ interface generated by the "direct oxidation" method described in Ref. [27]. Briefly, the structure consists of a silicon slab with an oxide layer "grown" on it in a QMD simulation. Si layers are separated from the stoichiometric oxide by approximately $4 \AA$ of sub-oxide. Structural models of that type are used in calculations of the band offset at the interface discussed in Section IV. We constructed several cells of various dimensions and thicknesses, with and without dangling bonds at the interface. Then, in the simplest case, a mirror image of the cell is generated, and the two are fused together. That procedure results in a $\mathrm{Si}-\mathrm{SiO}_{x}-\mathrm{SiO}_{2}-\mathrm{SiO}_{x}-\mathrm{Si}$ structure. Some special care needs to be taken of the $\mathrm{SiO}_{2}$ bonding pattern in the plane of contact. Mixing and matching the initial $\mathrm{Si}_{-} \mathrm{SiO}_{2}$ structures both symmetric and asymmetric MOS models could be built. The structure then is annelated in a high-temperature QMD run followed by a quench to guide the system to a local energy minimum. Models thus generated are used as scattering regions or "defects" in transport calculations of Section V. In particular we will describe the tunneling through a "defect" with the dimensions of $0.543 \mathrm{~nm} \times 0.543 \mathrm{~nm} \times 3 \mathrm{~nm}$. It consists of two monolayers of perfect (stoichiometric) $\mathrm{SiO}_{2}$, which are sandwiched between two $\mathrm{SiO}_{x}$ transition layers $0.3 \mathrm{~nm}$ each. This structure is embedded between two $1 \mathrm{~nm}$ thick regions of perfect silicon, and relaxed. The tunneling region is about $1.1 \mathrm{~nm}$. There are dangling bonds in one of the interfacial layers, while the other represents a perfect interface. Once we have a "defect" model, two perfect $\mathrm{Si}$ regions with the same cross section as the "defect" representing the leads are considered attached on both sides of the tunneling structure. Thus the total length of the system is taken to infinity. The left $\mathrm{Si}$ region represents the channel, and the right one a grain of poly-Si (gate electrode). The metallic nature of the gate electrode is taken into account by adjusting the Fermi level at the appropriate energy in the conduction band.

Silicon oxynitrides are considered leading candidates for an interim medium-k gate dielectric [28]. However, the properties of these materials are not very well understood. We investigate an oxynitride layer at the $\mathrm{Si}-\mathrm{SiO}_{2}$ interface and the effect of nitrogen on the band offset and leakage. In order to build a nitrided "defect" we first introduce nitrogen as nitric oxide NO at the Si$\mathrm{SiO}_{2}$ interface with the dangling bonds, and relax the structure. The details of these calculations, and the experimental verification of the proposed structures are reported in Ref. [29]. The total nitrogen concentration in the sample discussed in the following is $6.78 \times 10^{14} \mathrm{~cm}^{-2}$. The Si-SiON$\mathrm{SiO}_{2}$ structure is shown in Figure 1. Si-N bonds are ranging from 0.165 to $0.172 \mathrm{~nm}$, and there are no N-O bonds in the sample. All $\mathrm{N}$ atoms are three fold coordinated by $\mathrm{Si}$ just as in stoichiometric $\mathrm{Si}_{3} \mathrm{~N}_{4}$ or $\mathrm{Si}_{2} \mathrm{~N}_{2} \mathrm{O}$. The $\mathrm{Si}-\mathrm{N}-\mathrm{Si}$ bond angles are close to $120^{\circ}$. To build a tunneling "defect" structure we fuse this cell with a nitrogen free one with a perfect $\mathrm{Si}_{-} \mathrm{SiO}_{2}$ interface and relax the

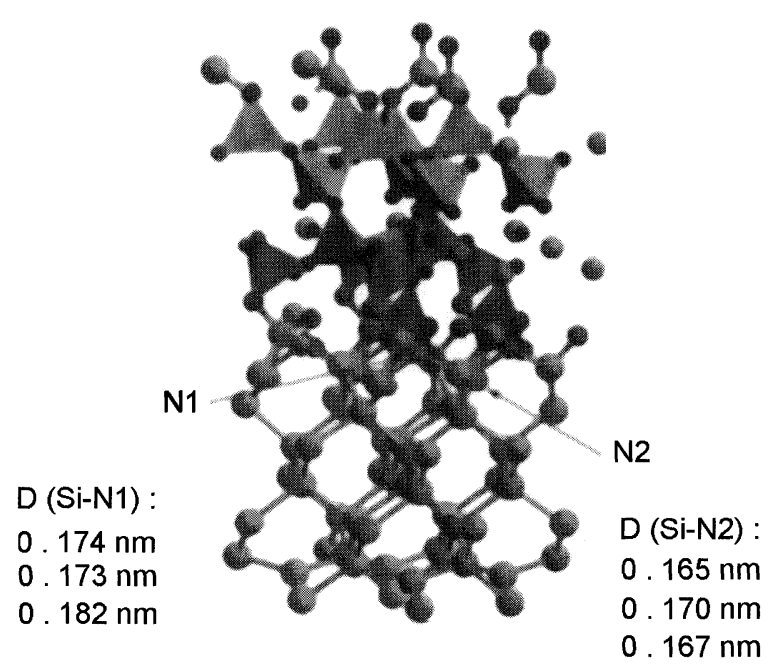

FIGURE 1 The Si-SiON-SiO 2 structure built in a Quantum Molecular Dynamics simulation. Nitrogen is at the interface where it is easier to realize the preferred three-fold coordination. 
local strain via a QMD anneal. The resulting asymmetric $\mathrm{Si}-\mathrm{SiON}-\mathrm{SiO}_{2}-\mathrm{SiO}_{x}-\mathrm{Si}$ structure has no dangling bonds, and the thickness of the tunneling region is similar to that of a nitrogen free sample described above.

To summarize, we have described a method to produce atomistic level models of MOS structures that could be used to calculate band offsets and transport properties.

\section{BAND OFFSET ESTIMATES}

We now briefly describe the "reference energy method" introduced in Ref. [27], and used here to estimate the valence band discontinuity at the interface. The technique is a local orbital formulation of the method due to Van de Walle and Martin [30]. We use the average of the expectation value of the Hamiltonian calculated for the valence (3s or $3 \mathrm{p}$ ) states of $\mathrm{Si}, \varepsilon_{s}(i)=H(s)_{\mathrm{aver}}$ and $\varepsilon_{p}(i)=H(p)_{\text {aver }}\left(H(s)=\left\langle\phi_{s}(i)|H| \phi_{s}(i)\right\rangle\right.$ and $H(p)=$ $\left\langle\phi_{p}(i)|H| \phi_{p}(i)\right\rangle$ respectively) as a reference energy against the valence band top of the bulk material. This is similar to a technique adopted in all-electron calculations for core level shifts [31]. The spacial dependence of $H(s)$ and $H(p)$ across a $20 \AA$ slab of silicon with two $2 \times 1$ reconstructed surfaces is shown in Figure 2. It is remarkable how fast both curves converge to their bulk values. In the following, we will use $\varepsilon_{s}$ as the reference energy.

The $\mathrm{Si} 3 \mathrm{~s}$ valence state matrix element $\mathrm{H}(\mathrm{s})$ plotted across the model heterojunction along the line perpendicular to the plane of the interface ( $\mathrm{Si}$ left, $\mathrm{SiO}_{2}$ right) is shown in Figure 3. Crosses correspond to the "as grown" sample, and diamonds correspond to the "annealed" sample. The interfacial region is clearly seen between -0.2 and $0.2 \mathrm{~nm}$. In $\mathrm{Si}$ (left), the average value of $\mathrm{H}(\mathrm{s})$ is near $-13 \mathrm{eV}$ while in the oxide (right) it is about $-20 \mathrm{eV}$. The valence band offset, $\Delta_{V B O}$, is obtained from

$$
\begin{aligned}
\Delta_{V B O}= & \Delta E_{R}\left(\mathrm{Si}, \mathrm{SiO}_{2}\right) \\
& +\Delta E_{R V}(\mathrm{Si})-\Delta E_{R V}\left(\mathrm{SiO}_{2}\right),
\end{aligned}
$$

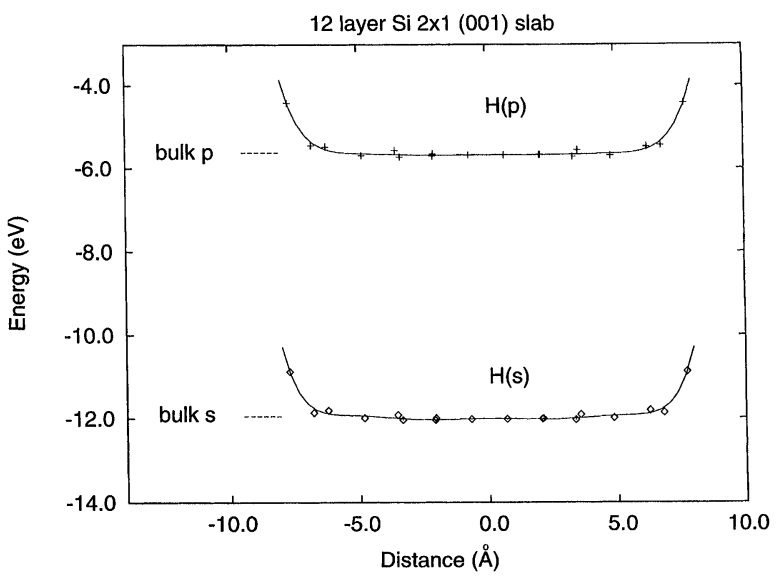

FIGURE 2 The spacial dependence of the $3 \mathrm{~s}$, and $3 \mathrm{p}$ expectation values of the Hamiltonian $H(s)=\left\langle\phi_{s}(i)|H| \phi_{s}(i)\right\rangle$ and $H(p)=\left\langle\phi_{p}(i)|H| \phi_{p}(i)\right\rangle$ across and $20 \AA$ slab of silicon with two $2 \times 1$ reconstructed surfaces.

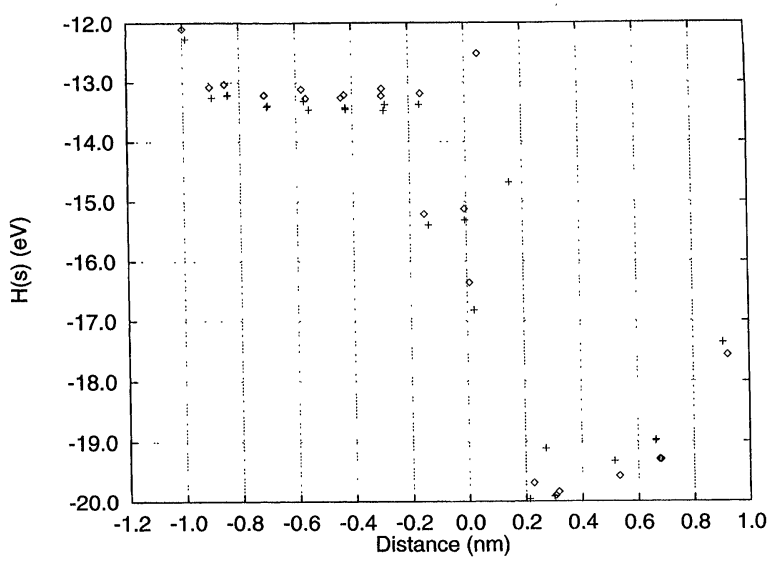

FIGURE 3 The $\mathrm{Si} 3 \mathrm{~s}$ valence state matrix element $H(s)$ plotted across the model heterojunction. Crosses correspond to the "as grown" sample, and diamonds correspond to the "annealed" sample. The interfacial region is clearly seen between -0.2 and $0.2 \mathrm{~nm}$.

where $\Delta E_{R}\left(\mathrm{Si}, \mathrm{SiO}_{2}\right)$ is the energy difference in the reference levels in $\mathrm{Si}$ and $\mathrm{SiO}_{2}$ respectively, and $\Delta E_{R \nu}(\mathrm{i})$ is the energy difference between $\varepsilon_{s}(i)$ and the top of the valence band edge in bulk material $i$. $\Delta E_{R V}(\mathrm{Si})$ is $8.31 \mathrm{eV}$. The value of $\Delta E_{R V}\left(\mathrm{SiO}_{2}\right)$ (which determines where to place the top of the valence band in the oxide layer) depends on $\mathrm{Si}-\mathrm{O}$ bond lengths and $\mathrm{Si}-\mathrm{O}-\mathrm{Si}$ bond angles. We estimate this dependence using $\beta$-cristobalite, and 
find that to first order the dependence on $\mathrm{Si}-\mathrm{O}-\mathrm{Si}$ angle is weak for angles in the range from $130^{\circ}$ to $180^{\circ}$ (where nearly all bond angles in silica polymorphs lie), and we shall neglect it in the analysis. In Figure 4 we show $\Delta \mathrm{E}_{R V}\left(\mathrm{SiO}_{2}\right)$ as a function of the bond length $\mathrm{d}_{\mathrm{SiO}}$. The dependence is well described by a linear equation:

$$
\Delta E_{R V}\left(\mathrm{SiO}_{2}\right)=-11.4 d_{\mathrm{SiO}} \mathrm{eV} / \AA+28.03 \mathrm{eV} .
$$

For the thin oxide layer we obtain a valence band offset of $4.35 \mathrm{eV}$, and for a thicker oxide layer we obtain $4.65 \mathrm{eV}$ in good agreement with experiment. In this procedure two quantities define the valence band offset. First, it is $\Delta E_{R}\left(\mathrm{Si}, \mathrm{SiO}_{2}\right)$, the difference in the Si reference energy level between the silicon and oxide layers. This difference takes into account the interfacial dipole. Secondly, it is $\Delta E_{R V}\left(\mathrm{SiO}_{2}\right)$ that is sensitive to the local strain in the oxide layer.

A more direct theoretical technique to estimate the valence band offset is the density of states analysis. We use the Green's function method within the local-orbital formalism to calculate the total and partial densities of states. The density of states is computed as:

$$
N(z)=-\frac{1}{\pi} \operatorname{Tr}\left(\operatorname{Im}\left(G(z) S^{-1}\right)\right)
$$

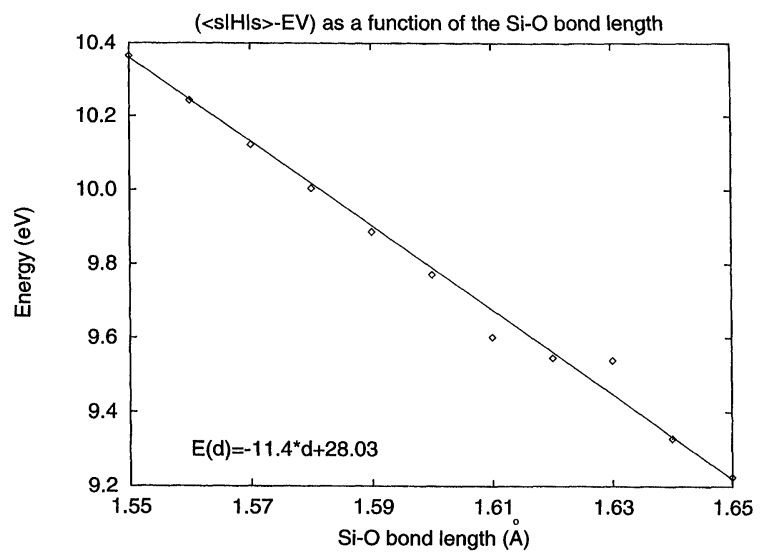

FIGURE $4 \Delta E_{R V}\left(\mathrm{SiO}_{2}\right)$ as a function of of the bond length $\mathrm{d}_{\text {SiO }}$ calculated for $\beta$-cristobalite exhibits a strong linear dependence. $\Delta E_{R V}\left(\mathrm{SiO}_{2}\right)$ is therefore sensitive to the local strain in the oxide layer. where $G(z)$ is the matrix of the resolvant operator $\mathbf{G}=(z-H)^{-1}$ in the local orbital basis not to be mixed with the representation of the resolvant in this basis. $S^{-1}$ is the inverse of the overlap matrix. The presence of the overlap is due to the nonorthogonality of the basis. The partial density of states is computed for the atoms in the different layers of the interfacial model and provides the energy-position correlation required for this analysis. The details of the calculation could be found in Ref. [29]. The valence band offset of $4.25 \mathrm{eV}$ is found in close agreement with the "reference energy method" analysis.

We repeated the analysis described above for the $\mathrm{N}$ containing sample. The valence band offset of $4.67 \mathrm{eV}$ is computed using the "reference energy method", and $4.7 \mathrm{eV}$ is obtained using the direct DOS analysis. The $0.32 \mathrm{eV}$ increase of the valence band offset after the nitridataion is mainly due to the strain relaxation caused by nitrogen in the interfacial layer $\left(\Delta E_{\mathrm{RV}}\left(\mathrm{SiO}_{2}\right)\right)$, only $0.12 \mathrm{eV}$ are coming from a change in $\Delta E_{R}\left(\mathrm{Si}, \mathrm{SiO}_{2}\right)$ that describes the interfacial dipole. Another potentially important result is a significant difference in the densities of states of two samples just below the $\mathrm{Si}$ conduction band edge. The partial density of states analysis indicates that the states in this energy region are due to the interface. The interface band is more pronounced in the nitrogencontaining sample, and is shifted down in energy by $0.3 \mathrm{eV}$. This possibly suggests that a very high nitrogen concentration at the $\mathrm{Si}-\mathrm{SiO}_{2}$ interface may result in the additional scattering due to the states just below the conduction band of $\mathrm{Si}$.

\section{TRANSPORT CALCULATIONS}

A detailed discription of the ballistic conductance calculation within a local orbital formalism will be reported separately [32]. Here we briefly outline the salient features of our approach. We identify the low-bias leakage through the ultrathin MOS structure with the carrier tunneling through a potential barrier caused by the band discontinuity 
at the silicon-dielectric interface. Therefore, we need to calculate the transmission probability for the electron traveling from the left lead to appear in the right lead after being "scattered" by a thin layer of the oxide or "defect". Mathematically, the problem is most conveniently dealt with within the framework of the scattering theory. In the eigenvector representation, the barrier transmission function is given by:

$$
T(E)=\sum_{l r}|\langle l|t| r\rangle|^{2} \delta\left(E-E_{l}\right) \delta\left(E-E_{r}\right),
$$

where $|l\rangle$ and $|r\rangle$ represent the unperturbed states of the semi-infinite leads without the coupling to the "defeact" (the choice of the "defect" layer will be discussed later), $\delta$ functions ensure the energy conservation, and $t$ represents the so-called $\mathrm{t}$ matrix which is defined as:

$$
t=V+V G V
$$

Here $V$ describes the interaction between the "defect" and the leads. This interaction could be written as

$$
V=V_{d l}+V_{d r}
$$

where $V_{d i}$ is the coupling between the "defect" and the leads. We use the short range basis orbitals, the cutoff radii for the $\mathrm{O}$ and $\mathrm{Si}$ orbitals are chosen to be $r_{c}=3.6$, and 5.0 Bohr, respectively, that results in a third nearest neighbor model for Si. We define the "defect" as the oxide layer sandwiched between two sub-oxide regions and four atomic layers of $\mathrm{Si}$ on each side. With this choice of a "defect" structure, it can be shown [32] that the transmission is given by:

$$
T=\operatorname{Tr}\left[\Gamma_{l} G \Gamma_{r} G^{+}\right]
$$

where $\Gamma_{i}$ is defined as:

$$
\Gamma_{i}=\sum_{i} V_{d i}|i\rangle\langle i| \delta\left(E-E_{i}\right) V_{d i}^{+}=V_{d i} \Delta_{i} V_{d i}^{+},
$$

and we used the following identity:

$$
\sum_{i} \delta\left(E-E_{i}\right)|i\rangle\langle i|=\delta\left(E-H_{i}\right)=\Delta_{i} .
$$

Note, that Eq. (9) is an operator expression independent of the basis. A very similar expression can be derived for the non-orthogonal basis [32]. One needs to keep in mind, however, that the trace in this case is defined as

$$
\operatorname{Tr}[O]=\sum_{\alpha}\left\langle\alpha\left|O S^{-1}\right| \alpha\right\rangle .
$$

Fortunately, because the "defect"-lead coupling matrix is so sparse, we are only interested in the sub-matrix $G_{d}$ of the total Green's function, describing the "defect". This portion of the Green's function can be written as:

$$
G_{d}=\frac{I}{E-H_{d}^{0}-\Sigma_{l}-\Sigma_{r}},
$$

where $H_{d}^{0}$ is the unperturbed "defect" Hamiltonian, and $\Sigma_{i}$ is the self energy describing the coupling of the "defect" to the leads:

$$
\Sigma_{i}=V_{d i}^{+} G_{i}^{0}(s) V_{d i}
$$

From Eq. (14) it follows that we only need to know the "surface" block $G_{i}^{0}$ of the Green's function matrix of a semi-infinite lead to compute the self-energy. In the non-orthogonal basis employed here, the Hamiltonian is a band matrix. Defining a slab as four atomic layers of $\mathrm{Si}$ we ensure that there is no interaction between the slabs beyond the nearest neighbour interaction. This results in an effective "tridiagonal" matrix similar to that for a 1D chain. Now the "surface" block is easily obtained by recursion. Also note, that by including a sufficient number of Si layers into the "defect" structure the coupling of the lead to the "defect" $V_{d i}$, is made essentially the same as the slab-slab coupling within the lead. The actual silicon-oxide coupling is "absorbed" in the "defect" Hamiltonian, and is treated exactly within the present formalism. 


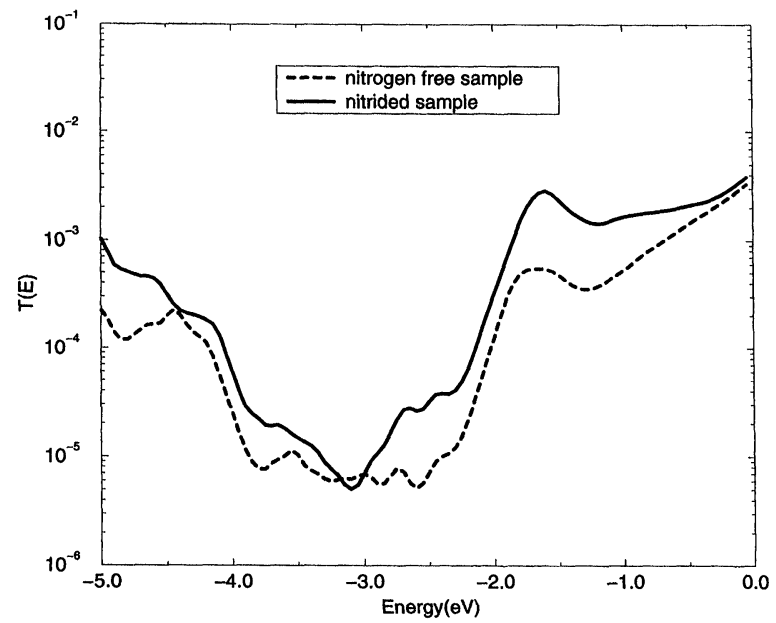

FIGURE 5 The transmission probability through two MOS structures. One is a nitrogen free sample with dangling bonds at the interface, and the other contains $6.78 \times 10^{14} \mathrm{~cm}^{-2}$ nitrogen at the silicon-silicon dioxide interface.

We have applied the described formalism to the gate leakage problem using our ultrathin MOS models as "defect" structures. In Figure 5 we show the transmission probability through two previously described MOS structures. One is a nitrogen free sample with dangling bonds at the interface, and the other contains $6.78 \times 10^{14} \mathrm{~cm}^{-2}$ nitrogen at the silicon-silicon dioxide interface. Note that the electron leakage is higher for the nitrogen-containing sample due to the increase in the density of states at the conduction band edge described in the previous section. That suggests that nitridation of the oxide layer may result in a higher leakage due to the segregation of nitrogen to the oxide-silicon interface in the case of high the nitrogen concentration.

\section{CONCLUSIONS}

We have described a theoretical approach suitable for screening of potential gate dielectrics. Using a combination of the density functional quantum molecular dynamics and ballistic transport theory we investigate the leakage current through ultrathin MOS structures. To our knowledge, this is the first entirely quantum mechanical approach to this problem. We believe that theoretical modeling could prove to be a useful tool in reducing the cost of the empirical search for a new gate dielectric.

\section{Acknowledgement}

It is our pleasure to thank D. Vasileska, R. Akis, D. Ferry, D. Drabold, J. Hallmark, R. Liu, and E. Hall for stimulating discussion and support of our work. X. Zhang and H. Loechelt thank Motorola, Inc. for support through the Motorola Summer Internship Program.

\section{References}

[1] 1999 International Technology Roadmap for Semiconductors, San Jose (1999).

[2] Muller, D. A., Sorsch, T., Moccio, S., Baumann, F. H., Evans-Lutterdodt, K. and Timp, G. (1999). Nature, 399, 758.

[3] Yu, P. Y. and Cardona, M., Fundamentals of Semiconductors, (Springer, New York, 1995) p. 325.

[4] McKee, R. A., Walker, F. J. and Chisholm, M. F. (1998). Phys. Rev. Lett., 81, 3014.

[5] Yu, Z., Droopad, R., Ramdani, J., Curless, J., Overgaard, C., Finder, J., Eisenbeiser, K., Wang, J., Hallmark, J. and Ooms, W., Mater. Res. Soc. Symp. Proc., 567, (Pittsbugh, PA 1999) 427.

[6] Eisenbeiser, K., Finder, J., Yu, Z., Ramdani, J., Curless, J., Hallmark, J., Droopad, R., Ooms, W., Salem, L., Bradshaw, S. and Overgaard, C. D. (2000). Applied. Phys. Lett., 76, 1324.

[7] Copel, M., Gribelyuk, M. and Gusev, E. (2000). Applied. Phys. Lett., 76, 436.

[8] Wilk, G. D. and Wallace, R. M. (1999). Applied. Phys. Lett., 73, 436.

[9] Kim, H. S., Gilmer, D. C., Campbell, S. A. and Polla, D. L. (1996). Applied. Phys. Lett., 69, 3860.

[10] Alers, G. B., Werder, D. J., Chabal, Y., Lu, H. C., Gusev, E. P., Garfunkel, E., Gustafsson, T. and Urdahl, R. S. (1998). Urdahl, Applied. Phys. Lett., 73, 1517.

[11] Robertson, J. (2000). J. of Vac. Sci. Technol. B, 18, 1785.

[12] Sirenko, A. A., Bernhardd, C., Golnik, A., Clark, A. M., Hao, J., Si, W. and Xi, X. X. (2000). Nature, 404, 373.

[13] Landauer, R. (1957). IBM. J. Res. Dev., 1, 223.

[14] Datta, S., Electronic Transport in Mesocopic Systems, (Cambridge, 1995).

[15] Porod, W. and Ferry, D. K. (1985). Phys. Rev. Lett., 54, 1189.

[16] Fischetti, M. V., DiMaria, D. J., Batey, J., Dory, L., Tierney, E. and Stasiak, J. (1987). Phys. Rev. B, 35, 4404.

[17] DiMaria, D. J., Fischetti, M. V., Batey, J., Dory, L., Tierney, E. and Stasiak, J. (1986). Phys. Rev. Lett., 57, 3213.

[18] Samanta, M. P., Tian, W., Datta, S., Hendderson, J. J. and Kubiak, C. P. (1986). Phys. Rev. B, 53, R 7626. 
[19] Shanware, A., Massoud, H. Z., Vogel, E., Henson, K., Houser, J. R. and Wortman, J. J. (1999). Microelectronic Engineering, 48, 295.

[20] Lucovsky, G. et al. (1998). J. of App. Phys., 83, 2327.

[21] Luttinger, J. M. and Kohn, W. (1955). Phys. Rev., 97, 869.

[22] Fisher, D. S. and Lee, P. A. (1981). Phys. Rev. B., 23, 6851.

[23] Haydock, R., Heine, V. and Kelly, M. J. (1978). J. Phys. $C, \mathbf{5}, 2848$.

[24] Demkov, A. A., Ortega, J., Sankey, O. F. and Grumbach, M. P. (1995). Phys. Rev. B, 52, 1618.

[25] Sankey, O. F. and Niklewski, D. J. (1989). Phys. Rev. B, 40, 3979.
[26] Sankey, O. F., Demkov, A. A., Windl, W., Fritsch, J. H., Lewis, J. P. and Fuentes Cabrera, M. (1998). Int. Journ. of Quant. Chem., 69, 327.

[27] Demkov, A. A. and Sankey, O. F. (1999). Phys. Rev. Lett., 83, 2038.

[28] Hegde, R. I., Maiti, B. and Tobin, P. J. (1997). J. Electrochem. Soc., 144, 1081.

[29] Demkov, A. A., Liu, R., Zhang, X. and Loechealt, H. (2000). J. of Vac. Sci. and Technol. B, 18, 2388.

[30] Van de Walle, C. G. and Martin, R. M. (1987). Phys. Rev. $B, 35,8154$.

[31] Picozzi, S., Massidda, S., Continenza, A. and Resta, R. (1997). Phys. Rev. B, 55, 16318.

[32] Zhang, X. et al., to be published. 

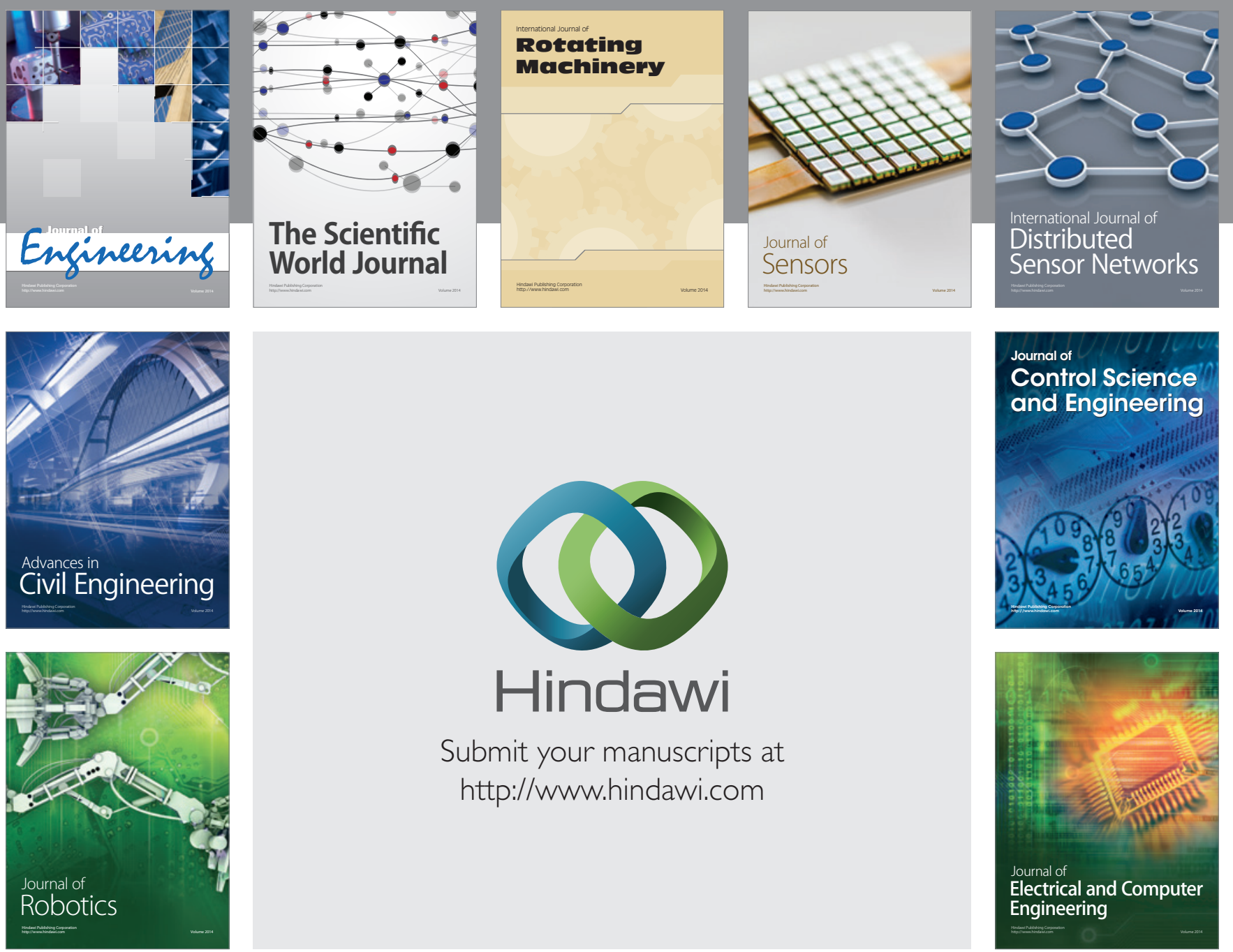

Submit your manuscripts at

http://www.hindawi.com
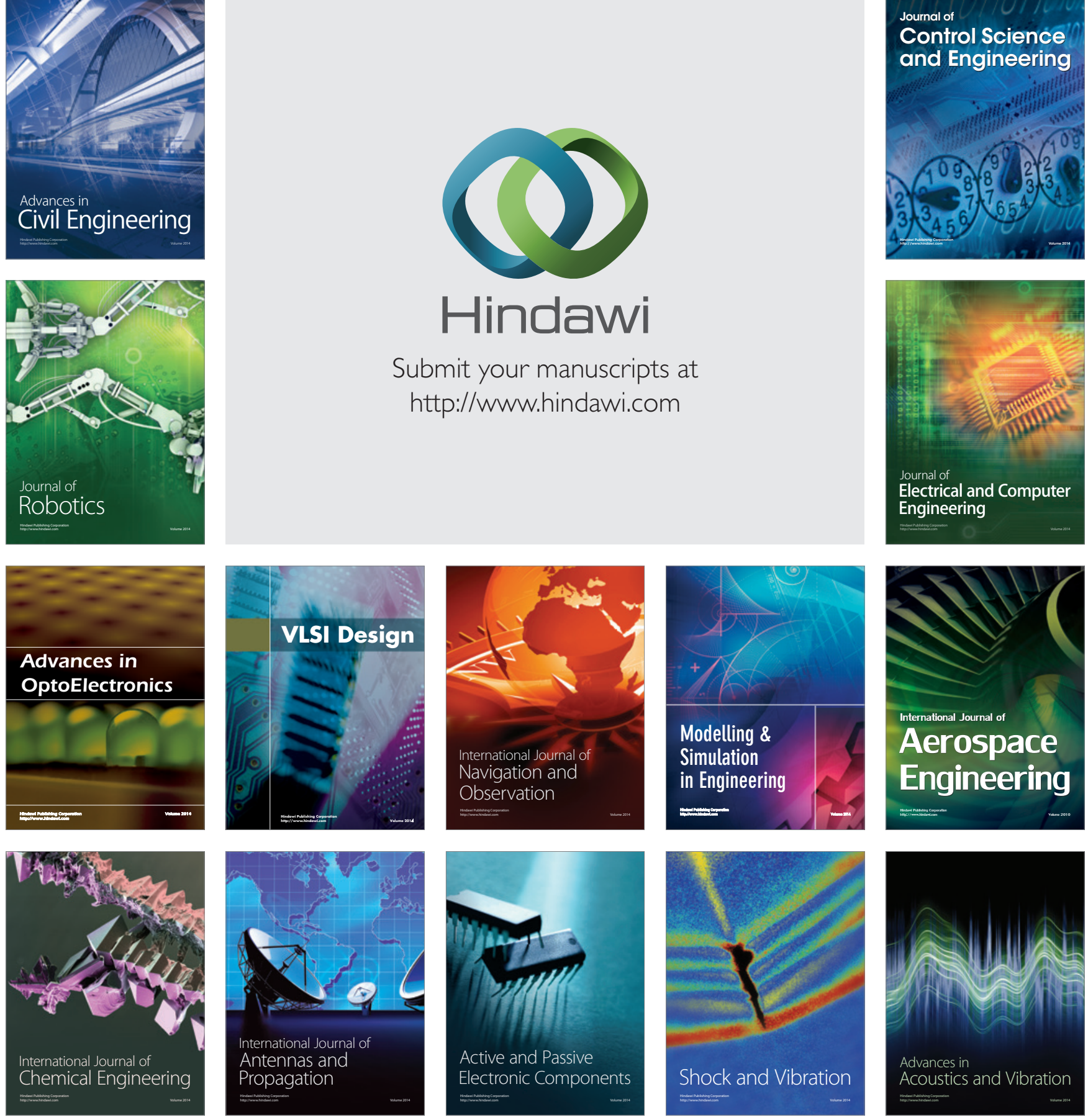